\title{
Reducing antibiotic costs: The Ottawa General Hospital experience of cost cutting, including intravenous to oral stepdown
}

\author{
GE GARBER MD FRCPC, W MCLEAN PharmD, M TIERNEY MSC, D PAGÉ MD FRCPC
}

$I^{*}$ N THE FISCAL YEAR 1991-92 THE MINISTRY OF HEALTH OF Ontario changed the rules for hospital funding, placing many hospitals in acute budgetary deficit. The Ottawa General Hospital, a 530-bed tertiary care institution, in anticipation of a shortfall in revenues, instructed the pharmacy department to cut $\$ 500,000$ from the medication budget or suffer a similar cut in staffing.

The antibiotic subcommittee of the pharmacotherapeutics committee at the hospital set up a plan for potential cost reduction in the area of antimicrobial therapeutics. The subcommittee, comprising members of the departments of pharmacy, microbiology, infectious diseases, hematology-oncology and surgery, developed a multifaceted plan for potential cost reduction. Initially, the most costly antimicrobials were identified. The six most costly anti-infective agents on hospital formulary included acyclovir, ceftazidime, clindamycin, imipenem, liposomal amphotericin $\beta$ and vancomycin. These agents were targeted for the development of strict 'Criteria for Use'. As well, guidelines for intravenous to oral conversion were adopted for the following medications: cefuroxime, ciprofloxacin, cotrimoxazole, erythromycin, fluconazole, metronidazole, as well as ranitidine and three corticosteroids. The dose interval of several antibiotics was extended based on updated literature review, including cefotaxime, ceftazidime, ceftizoxime, clindamycin, metronidazole, piperacillin and vancomycin.

Division of Infectious Diseases, Departments of Medicine and Pharmacy. Ottawa General Hospital, University of Ottawa, Ottawa, Ontario

Correspondence: Dr GE Garber, Division of Infectious Diseases, Ottawa General Hospital, 501 Smythe Road, Ottawa, Ontario K1H 8L6, telephone 613-737-8169, Fax 613-737-8099
As a final step a cost awareness program was initiated with a handbook provided to all members of the hospital housestaff showing comparative costs for commonly treated infections.

\section{RESULTS}

During the fiscal year 1992-93, \$582,000 was saved using this approach, with $\$ 340,000$ saved directly from the changes in protocols used for intravenous antimicrobial agents. This included decreasing the dose used of agents such as ceftazidime from $2 \mathrm{~g}$ q8h to $1 \mathrm{~g}$ q8h, changing the majority of acyclovir use on our Bone Marrow Transplant Service to oral instead of intravenous, and discouraging the use of vancomycin and imipenem unless clearly indicated. The intravenous to oral conversion saved close to $\$ 60,000$. Importantly, in the 1993-94 fiscal year no further growth in the drug budget has been seen, indicating that the program's success has been sustained despite a significant increase in acuity of illness in patients admitted to the hospital. Our infection control data indicate that there has been no increase in the incidence of nosocomial pneumonia, postoperative wound infections or line sepsis in our hospital.

In summary, our hospital has been able to achieve a significant cost savings with no perceived adverse outcome on patient care. This increased efficiency has been attained because of a collaborative and consultative approach between the pharmacy department, attending physicians and the consulting infectious disease and microbiology staff. Many of these sustainable cost saving measures outlined above have recently been adopted by other hospitals in our area. 


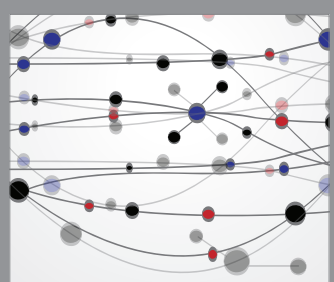

The Scientific World Journal
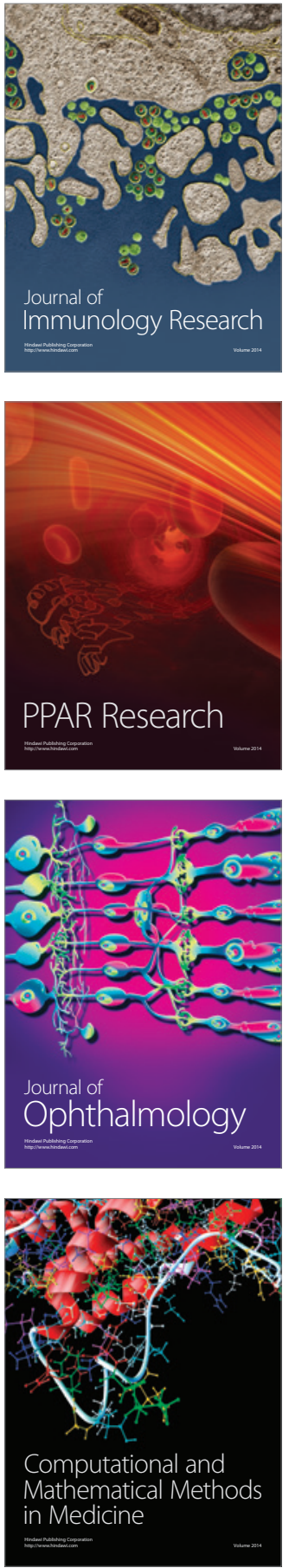

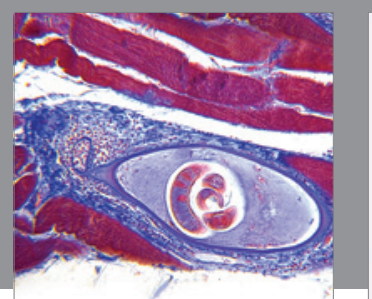

Gastroenterology Research and Practice

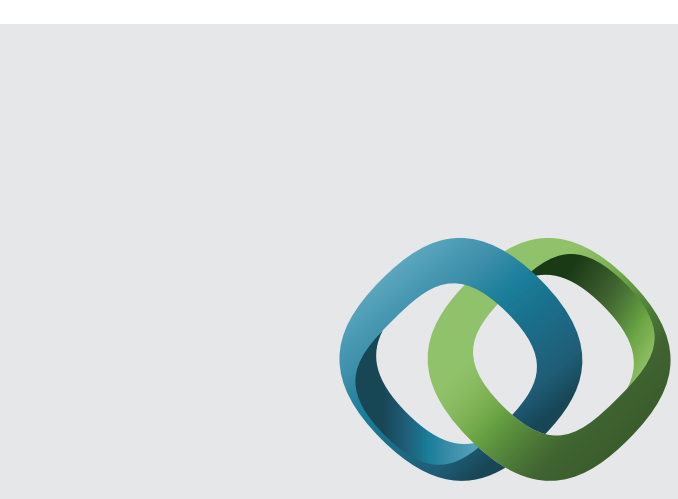

\section{Hindawi}

Submit your manuscripts at

http://www.hindawi.com
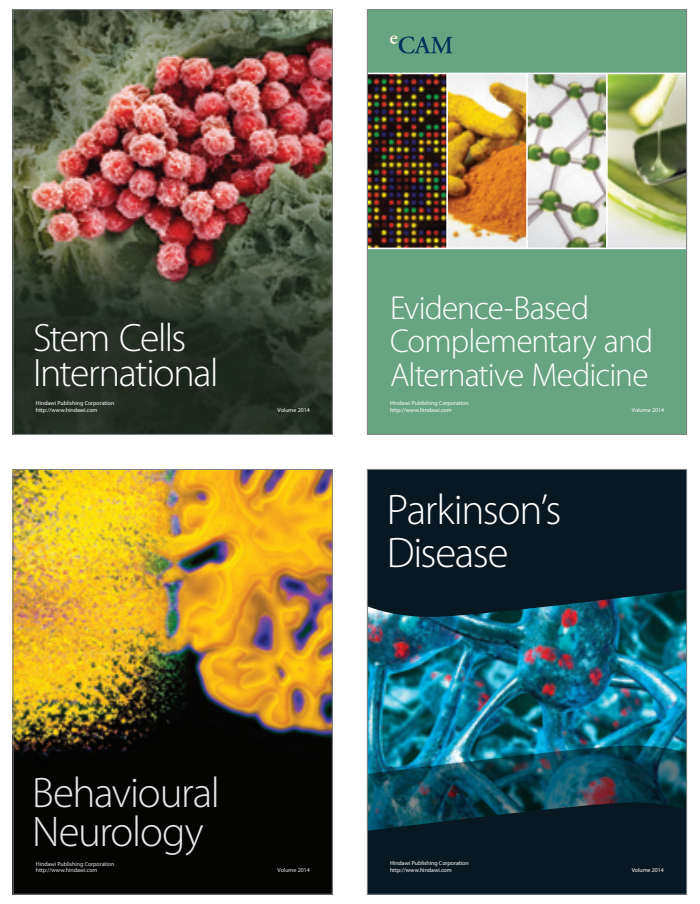
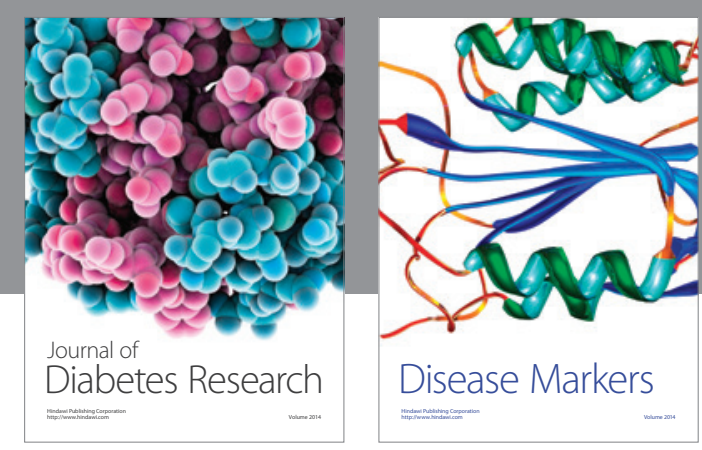

Disease Markers
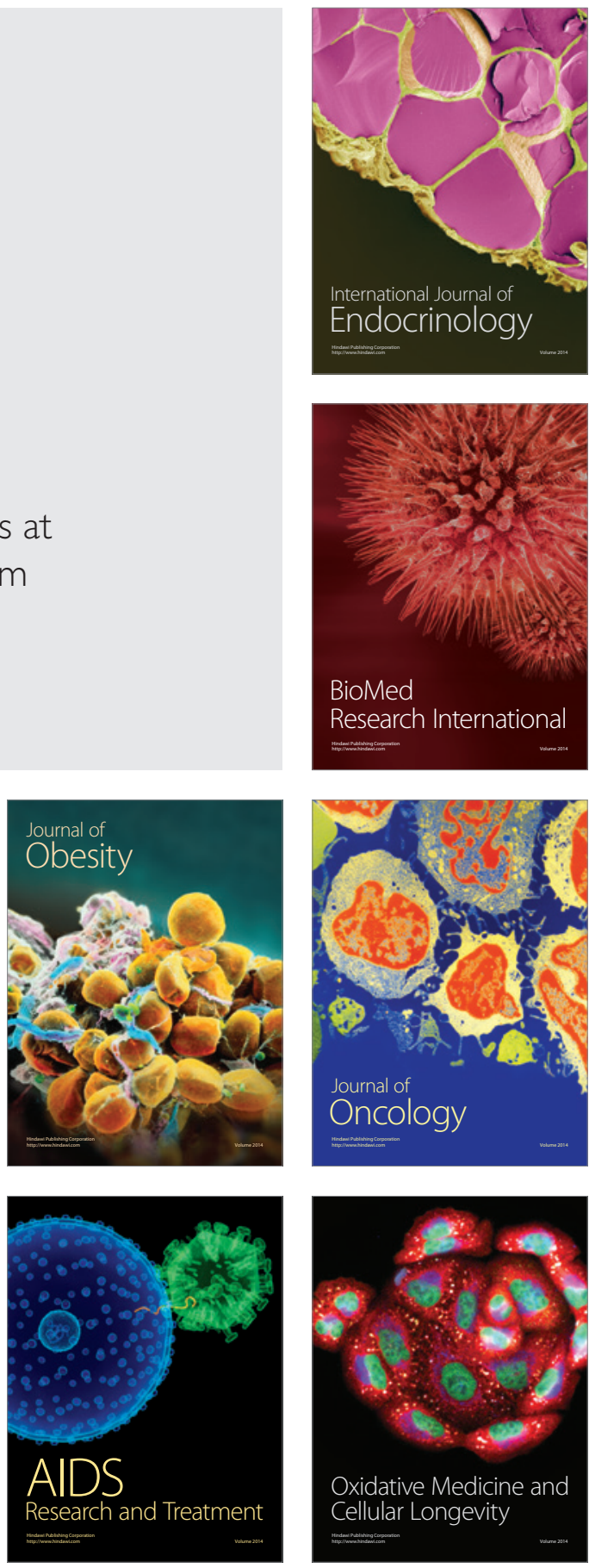